\title{
Auto Graph Encoder-Decoder for Neural Network Pruning
}

\author{
Sixing Yu \\ Iowa State University \\ yusx@iastate.edu
}

\author{
Arya Mazaheri \\ Technical University of Darmstadt \\ arya.mazaheri@tu-darmstadt.de
}

\author{
Ali Jannesari \\ Iowa State University \\ jannesarieiastate.edu
}

\begin{abstract}
Model compression aims to deploy deep neural networks $(D N N)$ on mobile devices with limited computing and storage resources. However, most of the existing model compression methods rely on manually defined rules, which require domain expertise. DNNs are essentially computational graphs, which contain rich structural information. In this paper, we aim to find a suitable compression policy from DNNs' structural information. We propose an automatic graph encoder-decoder model compression (AGMC) method combined with graph neural networks (GNN) and reinforcement learning $(R L)$. We model the target $D N N$ as a graph and use GNN to learn the DNN's embeddings automatically. We compared our method with rule-based DNN embedding model compression methods to show the effectiveness of our method. Results show that our learningbased DNN embedding achieves better performance and a higher compression ratio with fewer search steps. We evaluated our method on over-parameterized and mobile-friendly DNNs and compared our method with handcrafted and learning-based model compression approaches. On over parameterized DNNs, such as ResNet-56, our method outperformed handcrafted and learning-based methods with $4.36 \%$ and $2.56 \%$ higher accuracy, respectively. Furthermore, on MobileNet-v2, we achieved a higher compression ratio than state-of-the-art methods with just $0.93 \%$ accuracy loss.
\end{abstract}

\section{Introduction}

With the increasing demand to deploy deep neural networks (DNNs) on edge devices (e.g., mobile phones, robots, self-driving cars, etc.), which usually have limited storage and computing power, model compression techniques became essential for efficient DNN deployment. Network pruning $[10,11,32]$, factorization [46, 38], knowledge distillation [36, 34, 16], and parameter quantization [10, 49, 19] are among the most well-known model compression techniques. However, these methods heavily rely on handcrafted rules defined by experts, demanding an extensive amount of time and might not necessarily lead to a fully compressed model.

Recently, automatic model compression [14, 49, 27] has gained momentum. For example, Wang et al. [49] proposed a Bayesian automatic model compression method trained in a one-shot manner to find reasonable quantization policies. He et al. [14] proposed an automatic model compression method based on reinforcement learning (RL). However, when representing DNNs, they rely on manually defined DNN embedding vector (e.g., using one-hot vectors to characterize DNN's hidden layers) and ignore the rich structural information between the hidden layers.

DNNs are essentially represented as computational graphs in deep learning frameworks, such as TensorFlow [1] and PyTorch [35]. A computational graph is composed of numerous primitive operations (e.g., add, minus, multiply), where edges are operations and nodes are intermediate calculation results (i.e., feature maps in DNNs). Such a rich structural representation can effectively delineate the state of DNN hidden layers. Additionally, computational graphs often contain repetitive structural patterns due to the same set of primitive operations being used multiple times. Thus, we aim to benefit from this feature by extracting the structural information readily available within computational graphs to identify the redundancy and pruning policy for DNN hidden layers.

In this paper, we propose a graph-based Auto Graph encoder-decoder Model Compression (AGMC) method that combines graph convolutional networks (GCNs) [21, 53, 52] and reinforcement learning (RL) [25, 45, 43] to learn the compression strategy of DNNs without expert knowledge. The graph encoder-decoder aims to learn the DNN's layer embeddings. The GCN-based graph encoder learns the DNN representation from its structure information, and the decoder decodes the representation to hidden layer embeddings. The RL agent takes the hidden layer embeddings as the environment states, looks for the pruning ratio for each hidden layer, and generates a corresponding compressed candidate model. Finally, we evaluate the candidate compressed model performance and provide a reward value as feedback to the RL agent. By exploiting DNN's struc- 
ture information to suggest compression policies, our approach successfully applied network pruning and achieved outstanding results on various DNNs, such as ResNet [12], VGG-16 [44], MobileNet [17, 39], and ShuffleNet [54, 29].

In essence, this paper makes the following contributions:

- A novel automatic layer embedding based on computational graph's structure.

- An efficient method based on GCN and RL to automate the channel pruning.

- State-of-the-art model pruning results on various DNN models.

\section{Background and related work}

With the increasing demand to make edge devices smarter using AI, efficient deep neural network design is more important than ever. Hence, various efficient networks have been introduced to reduce the computational complexity and memory requirement of such networks. MobileNetv1/v2 [17, 39], ShuffleNet-v1/v2 [54, 29], DiCENet [31], and CondenseNet [18] are among the notable efforts that introduced custom convolutional blocks to improve the overall efficiency. Furthermore, neural architecture search (NAS) [55, 6, 4, 30] methods also attempt to generate efficient DNNs by searching for the most optimal neural network structure, given the constraints of the target hardware platform.

Within the context of this paper, we discuss former studies related to model compression, particularly network pruning and the application of GCN in model compression. In the following, we will provide a brief overview of these methods.

Model compression. A multitude of previous work focus on model compression techniques, such as knowledge distillation [36, 34, 16], parameter quantization [10, 49, 19], factorization [46, 38], and network pruning [10, 11, 32].

As DNNs are typically over-parameterized, network pruning is among the most widely used model compression techniques, which has achieved outstanding results and can dramatically shrink model size [2]. It eliminates a portion of parameters and computation within each DNN layer via two different methods: (1) fine-grained pruning and (2) structured pruning. Fine-grained pruning [10] targets individual unimportant elements in weight tensors. On the other hand, structured pruning [24] attempts to prune entire blocks of weight tensors, such as channels, rows, columns, and blocks. Although the fine-grained pruning could achieve a high compression rate with minimal accuracy loss, they lead to irregular sparsity patterns, demanding specialized hardware accelerators [20,9] to realize any speedup. Alternatively, using structured pruning results in regularly pruned weights and can be used on commodity hardware. In this paper, we particularly focus on structured pruning.

Empirical pruning policies are uniform, shallow, and deep [15, 24]. The uniform policy sets the compression ratio uniformly. The shallow and deep policies aggressively prune shallow and deep layers, respectively. Such handcrafted empirical policies heavily rely on manually defined rules and might not lead to an optimal compression policy. Other handcrafted methods focusing on channel pruning are SPP[48], FP [24], and RNP [26]. SPP prunes DNNs by analyzing each layer and measures the reconstruction error to determine the pruning ratios. FP evaluates the performance of single-layer pruning and estimates the sensitivity of each layer. Layers with lower sensitivity are pruned more aggressively. RNP introduced an RL-based method and groups all convolutional channels into four sets for training.

Conventional network pruning methods primarily rely on handcrafted and rule-based policies, demanding human effort and domain expertise. Moreover, such methods might not necessarily offer a fully compressed model. Recently, RL-based automatic network pruning methods [51, 14, 27] have been proposed. Liu et al. [27] proposed an ADMMbased [3] structured weight pruning method and an innovative additional purification step for further weight reduction. He et al. [14] proposed AutoML for network pruning, which leverages RL to predict compression policies, yet they still use handcrafted rules (11 fixed features) to represent DNNs and ignore the rich structural information within computational graphs.

Graph neural networks. GNN and its variants [22, 40] are successfully applied to learn the topology information from graphs. For instance, they have been successfully applied to node classification, link prediction, and graph classification. Furthermore, graph-based NAS methods [8, 42, 5] model DNNs as computational graphs and find the optimal DNN structure from a graph-based search space. These methods inspired us to use a GCN-based graph encoder to learn the DNN embeddings.

\section{Methodology}

To prune a given DNN, we first modeled the DNN as a computational graph and introduced a GCN-based graph encoder to learn the DNN's representation $g$. Then the decoder decodes the $g$ in to layer embeddings $s_{i} \in S, i=$ $1,2, . ., T$, where $T$ is the number of hidden layers. Since we aim to compress the DNN by predicting the pruning ratio for each hidden layer, the RL agent takes the layer state $S$ as the environment state to search for the hidden layer's pruning policy $a_{i} \in A, i=1,2, . ., T$. The pruned DNN's performance is then used as a reward for the current actions $A$ taken by the RL agent.

Figure 1 depicts an overview of our method. In the following, we will explain the details of the simplified computa- 


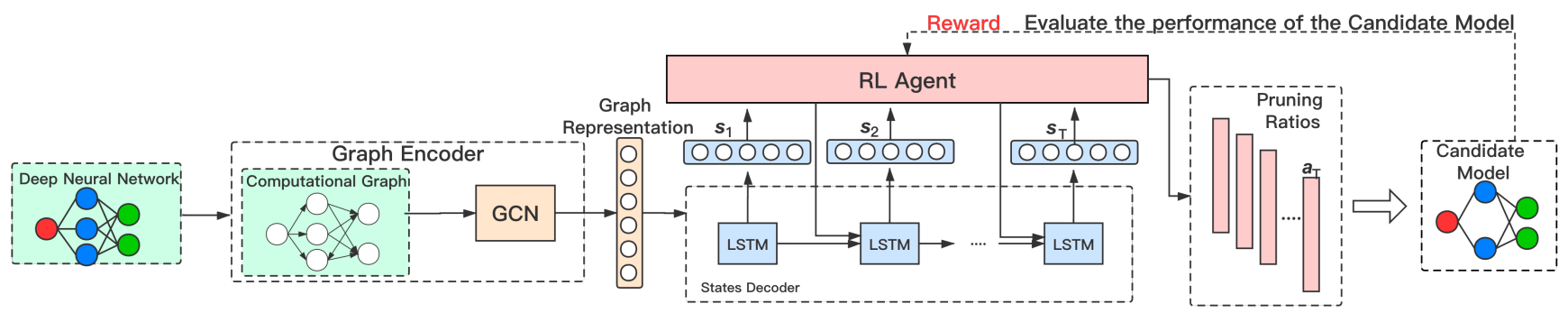

Figure 1. The workflow of Auto Graph encoder-decoder Model Compression (AGMC)

tional graph, graph encoder-decoder, and RL agent within our approach.

\subsection{Simplified graph representation of DNNs}

The computational graph representation of DNNs is composed of numerous primitive operations (e.g., add, minus, multiply), where edges are operations and nodes are intermediate results (i.e., feature maps). Thus, a typical computational graph might involve billions of primitive operations [12], making it unrealistic to use the graph for our analysis directly. To simplify the graph representation, we choose commonly used machine learning operations as primitive operations $\mathcal{O}=\{n \times n$ conv, Relu, BatchNorm, (Max/Average) Pooling, Padding, Splitting $\}$. Such a simplification can significantly reduce the graph complexity and yet preserve important structural information.

Formally, We model a given DNN as a single-source and single-sink computational graph $G=(V, E, \mathcal{O})$, where $V$ is the node-set, $E$ is the edge set, and $\mathcal{O}$ is the primitive operation set. Each directed edge with an edge type is associated with a primitive operation in $\mathcal{O}$. Figure 2 (a) shows the idea behind the simplified computational graph using two primitive operations $\mathcal{O}=\{1 \times 1$ conv, $3 \times 3$ conv $\}$, which correspond to two edge types. The computation graph $G$ denotes a compound operation composed of the primitive operations in $\mathcal{O}$ :

$$
y=\operatorname{assemble}(\operatorname{conv} 3(\operatorname{conv} 1(x)), \operatorname{conv} 3(\operatorname{conv} 1(x)))
$$

Figure 2 (b) shows another example for constructing a computational graph for a ResNet block, which contains a $1 \times 1$ convolutional layer with four output channels and a $3 \times 3$ convolutional layer with three output channels. Although different layers have different computational graphs, they often share similar structures.

\subsection{Auto graph encoder-decoder}

We introduce a GCN-based graph encoder-decoder to learn the embeddings of the target DNN's hidden layers automatically. The GCN-based graph encoder embeds the graph and learns the DNN's structure representation $g \in$ $\mathbb{R}^{1 \times d}$, where $d$ is the embedding size. We also introduced an LSTM [47] based decoder that decodes the DNN's representation to layer embeddings $S \in \mathbb{R}^{T \times d}$, where $N$ is the number of hidden layers.

\subsubsection{GCN-based graph encoder}

The GCN embeds graphs by aggregating node features from neighbor nodes. The message passing function can be formulated as follows:

$$
h_{i}^{l+1}=\sum_{j \in N_{i}} \frac{1}{c_{i}} W^{l} h_{j}^{l},
$$

where $h_{i}^{l}$ is the hidden state of $i^{\text {th }}$ node in GCN's $l^{\text {th }}$ convolution, $c_{i}$ is a constant coefficient, $N_{i}$ is node $i$ neighbors, and $W^{l}$ is GNN's learnable weight matrix.

Although standard GCN and its variants aim to learn the node embeddings in a graph, we aim to learn the entire graph representation. Thus we need to take the graph representation from the node embeddings. One of the most commonly used mechanisms to achieve this is to use the graph mean pool (Equation 4), which averages the node embeddings. The graph encoder is formulated as Equation 3. It embeds the computational graph and gets the node-embedding matrix $H$. Then, the graph mean pool reads the graph representation $g$ from the node embeddings.

$$
\begin{gathered}
H=\operatorname{GCN}_{\text {encoder }}(G) \in \mathbb{R}^{N \times d}, \\
g=\frac{1}{N} \sum_{i=1}^{N} h_{i},
\end{gathered}
$$

where $H=h_{i}, i=1,2, \ldots, N$ is the node-embedding matrix, $h_{i}$ is the embedding of $i^{t h}$ node, $N$ is the total number of nodes in the graph, and $d$ is the embedding size.

\subsubsection{Decoder}

The decoder aims to learn the environment states of DNN hidden layers for the RL agent. Since the state vectors in the $\mathrm{RL}$ environment are determined by the previous state and 


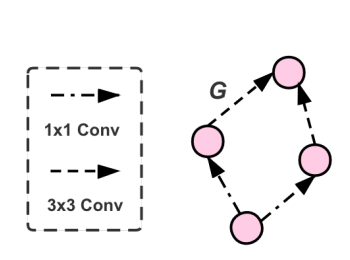

(a)
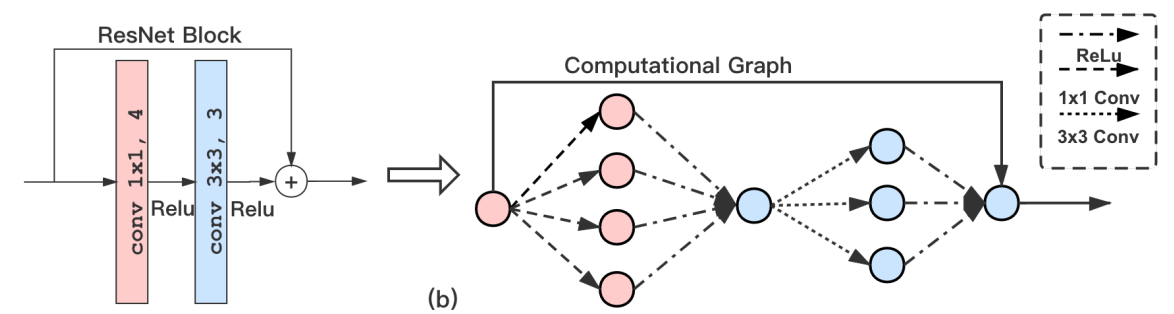

Figure 2. Simplified computational graph. (a) An example of a simplified computational graph. (b) Construction of a simplified computational graph for a ResNet block [12]

the action (the pruning ratio), the decoder takes the previous layer's states vector and RL agent's action as input:

$$
\begin{gathered}
s_{1}=\operatorname{LSTM}_{\text {decoder }}(g), \\
s_{t}=\operatorname{LSTM}_{\text {decoder }}\left(s_{t-1}, a_{t-1}\right)
\end{gathered}
$$

For the $t$-th hidden layer, we use the feature $s_{t-1}$ of the previous hidden layer and the compression policy $a_{t-1}$ (the action selected by the RL agent) to calculate the environment states.

\subsection{Automatic network pruning using reinforce- ment learning}

We leverage reinforcement learning to find the optimal pruning ratios efficiently. In the following, we describe the details of our reinforcement learning setup.

Environment states. In contrast to existing RL-based model compression methods that use fixed handcrafted layer embeddings as environment states, we use DNN layer embeddings $S \in \mathbb{R}^{T \times 1 \times d}$ generated by the graph encoderdecoder as environment states.

Action space. The actions made by the RL agent are pruning ratios within a continuous space. Specifically, the RL agent takes the layer embeddings $S \in \mathbb{R}^{T \times d}$ as environment states and predicts corresponding pruning ratios $a_{i} \in A, i=1,2, . ., T$, where $a_{i} \in[0,1)$.

Reward function. We prune the DNN according to the pruning ratio made by RL agent, and use the performance of the compressed model as the reward. The reward function is defined in Equation 7.

$$
R_{\text {err }}=- \text { Error }
$$

where Error is the compressed DNN's top-1 error on the validation set.
Deep deterministic policy gradient (DDPG). Various RL policies aim to search within a continuous action space, such as proximal policy optimization (PPO) [41] and deep deterministic policy gradient (DDPG) [25]. Similar to the AMC [14] method, we opted for DDPG as the RL policy to make a fair comparison and exclude the influence of RL policy on the experimental results. This way we can demonstrate the superiority of our learning-based embedding compared to the handcrafted rules.

The DDPG agent's search process can be formulated as following:

$$
\begin{gathered}
g \in \mathbb{R}^{1 \times d}=\text { GraphEncoder }(G), \\
S \in \mathbb{R}^{T \times 1 \times d}=\operatorname{Decoder}(g), \\
A \in \mathbb{R}^{T \times 1 \times 1}=\operatorname{MLP}(S),
\end{gathered}
$$

where $G$ is the computational graph, $g$ is the graph representation, $S$ is the environment states, and MLP is a multilayer perceptron neural network. The graph encoder embeds the graph $G$ and learns the DNN representation $g$ and the decoder decodes $g$ into hidden layers embeddings $s_{i} \in S, i=1,2, . ., T$. Finally, the RL agent takes $S$ as environment states and uses MLP to project the embedding into hidden layer pruning ratios $a_{i} \in A, i=1,2, . ., T$.

\subsubsection{Action rescaling}

The reward function that we use offers small or no incentive for model size and FLOPs reduction. Without any constraint (e.g., FLOPs or \#parameters), the RL agent tends to search for a tiny compression ratio. Thus, to obtain the desired model size reduction, we apply Algorithm 1 to adjust the action space $a$.

In essence, Algorithm 1 computes the size we still needed to reduce according to the original scale. Lines 1-2 compute the total model size (e.g., FLOPs and \#parameters) $W_{\text {all }}$ and reduced size $W_{\text {reduced }}$. If the reduced size is less than the desired model size reduction $d$, the algorithm will rescale the pruning ratios to compensate the difference 
$d-W_{\text {reduced }}$. Lines 4-7 relate to the rescaling process, and the for-loop in lines 5-7 adjusts the pruning ratio for each layer according to the difference to the desired model size reduction. Finally, in line 7, we truncate the pruning ratio with the upper bound $a_{\max }$.

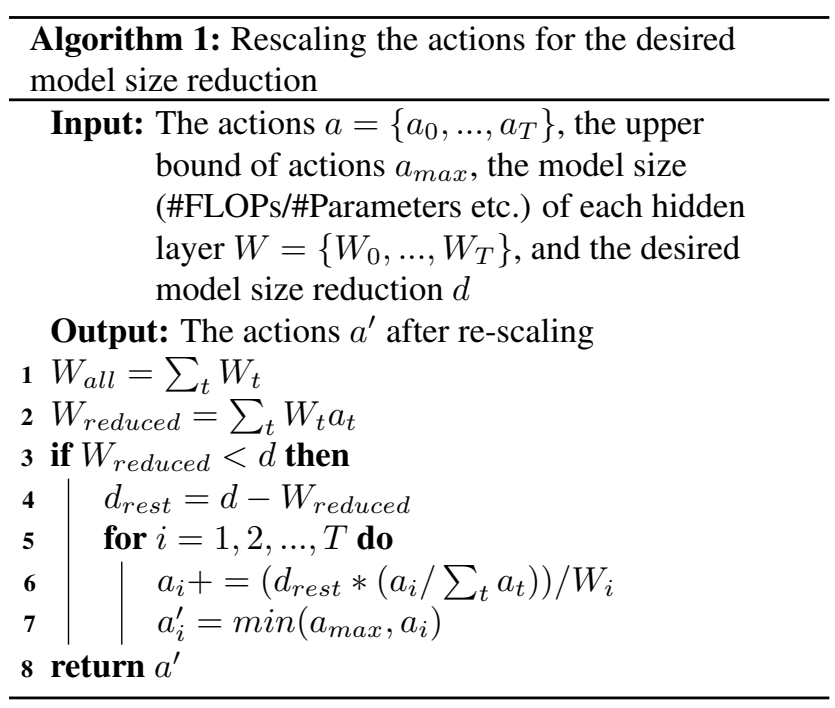

\section{Experimental results}

We evaluate our approach by performing FLOPsconstrained structured pruning on several convolutional networks, including over-parameterized DNNs (e.g., ResNet20/56 [12] and VGG-16 [44]) and mobile-friendly DNNs (e.g., MobileNet-v1/v2 [17, 39] and ShuffelNet$\mathrm{v} 1 / \mathrm{v} 2[54,29])$. To show the superiority of our approach, we compared our approach with various existing methods in different categories, such as:

- Uniform, shallow, and deep empirical policies [15, 24].

- Handcrafted channel reduction methods, such as SPP [48], FP [24], and RNP [26].

- Regularization-based methods, such as MorphNet [7] and SSL [50].

- RL-Based AutoML methods, such as auto model compression method AMC [14], which manually defines DNN layer embeddings, and random search with reinforcement learning (RS), which does not leverage any layer embeddings.

- Other pruning methods, such as DSA [33] and Rethink [28].

Finally, we show the inference acceleration and memory saving of compressed models on a GPU platform.
RL setup. The actor network $\mu$ and the critic network $Q$ have two hidden layers, each with 300 units. The $\mu$ 's output layer applies the sigmoid function to bound the actions within $(0,1)$. We use $\tau=0.01$ for the soft target updates. In the first 25 episodes, our agent searches with random action. Then, it continues searching for 300 episodes with exponential decayed noise. The graph encoder is a two-layer GCN with a hidden feature size of 50 units and a DNN embedding size of 11 units.

Datasets. We conducted our experiments using CIFAR10 [23], CIFAR-100 [23] and ILSVRC-2012 (ImageNet) [37] datasets. To accelerate the search process on CIFAR-10/100, we split the training set to $15 K$ and $5 K$ images. We used the $15 K$ training set to rapidly fine-tune the candidate model and the remaining $5 K$ images as the validation set to calculate the reward function. In the ILSVRC2012 dataset, we split $5 K$ images from the training set as the validation set to calculate the reward. The Validation accuracy of the ILSVRC-2012 dataset is very sensitive to the compression, as with high compression ratios, the accuracy drops considerably without fine-tuning. Thus, the RL agent can not get a valuable reward. As a remedy, we decompose the pruning on the ILSVRC-2012 dataset into several stages and add one epoch of fine-tuning for each search episode. For instance, to obtain a $49 \%$ FLOPs model compared to the original network, instead of performing a single step $49 \%$ FLOPs pruning, we prune the target DNN two times, each with $70 \%$ FLOPs constraint (i.e., 70\%FLOPs $\times 70 \%$ FLOPs $=49 \%$ FLOPs).

\subsection{The effectiveness of DNN embeddings}

In contrast to existing methods [27, 14], layer embeddings are essential for our learning-based automatic network pruning method. In the following, we analytically compare the effectiveness of using our DNN graph embedding with existing approaches.

Learning-based vs. manually-defined layer embeddings. We compare AGMC with AMC [14], which manually defines 11 features related to each layer as the embedding vector $s_{t}=$ $\left(t, n, c, h, w\right.$, stride, $k, F L O P s(t)$, reduced, rest,$\left.a_{t-1}\right)$ , where $t$ is the layer id, the dimension of the kernel is $n \times c \times k \times k$, and the input is $c \times h \times w . F L O P s(t)$ is the FLOPs of layer $t$. Reduced is the total number of FLOPs reduced in previous layers and finally rest is the number of remaining FLOPs in the layers ahead. We argue that such a strict layer embedding may miss important information, such as the number of parameters in each hidden layer, which are only applicable to a given DNN. In the AGMC, the graph encoder-decoder learns the layer states from DNN structural information. Thus, it does not 


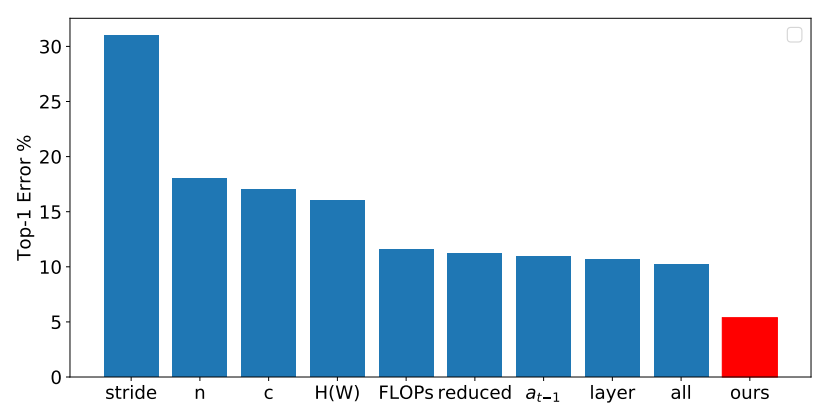

Figure 3. An error-rate comparison for individual AMC layer embedding, overall AMC, and AGMC layer embedding. Our method has achieved roughly $2 \times$ less error rate.

require expert knowledge and is applicable for all kinds of DNNs.

Since AMC has defined 11 features to represent a convolutional layer, we also set the learning-based embedding size to 11 (i.e., $S \in \mathbb{R}^{T \times 11}$ ). We evaluated our learningbased embeddings and the AMC handcrafted embedding on ResNet-20 pre-trained with the CIFAR-10 dataset. Figure 3 shows the spatial decomposition evaluation for the AMC's layer embeddings under 50\% FLOPs ResNet-20. Using stride as the only layer embedding, we get an error rate of $31 \%$ since it is difficult to distinguish different layers. However, combining the stride with the number of filters $n$ decreases the error rate to $18 \%$. Consequently, combining all the features leads to $10.2 \%$ error margin. On the other hand, our learning-based layer embedding achieves an error rate of $5.38 \%$, outperforming the manually defined layer embedding by a factor of two.

Learning-based vs. no embedding. We compared AGMC with random search (RS) without layer embedding. We set all the hidden layers of the RS setup to a fixed onehot vector as the RL agent's environment state and use $R_{\text {err }}$ as a reward. Then, we leverage the DDPG reinforcement learning agent to search pruning ratios for ResNet-20/56. ResNets have residual connections between their blocks, which instructs equal channel size between residual connection blocks. We opted for removing all the residual connections to avoid sharing the pruning ratios between residual connected layers and learn each hidden layer's embedding independently. As shown in Figure 4, AGMC achieves better results compared with RS on ResNet-20. Particularly, AGMC enabled us to find the compressed model with fewer episodes, higher accuracy, and more FLOPs reduction. Moreover, by further layer-wise analysis, we observed that AGMC tends to prune each layer uniformly and the pruning ratio is more stable than the RS. Such an observation is in line with the uniform pruning policy[24], which argues that the uniform policy can yield better pruning.

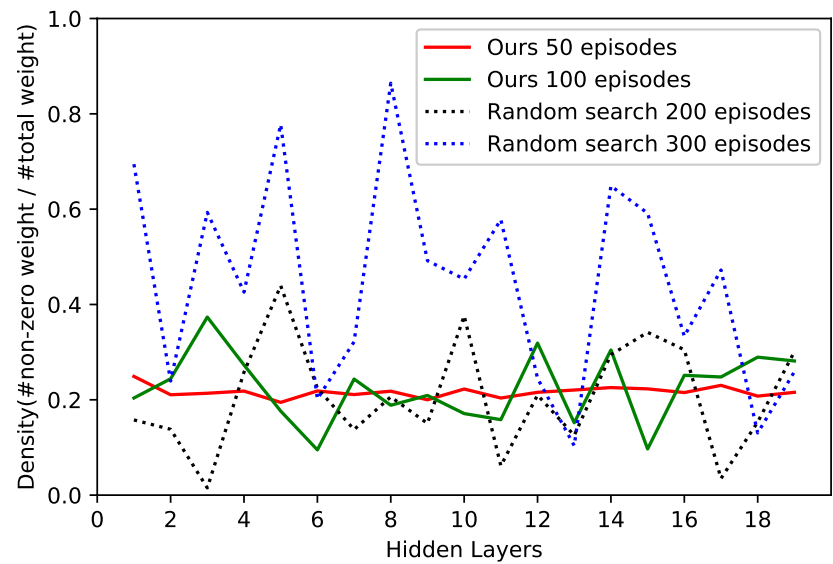

Figure 4. Comparing AGMC pruning stability across different layers with random search on ResNet-20. Random search uses 200 and 300 episodes, achieving a compressed network with $71 \%$ and $88.41 \%$ validation accuracy, respectively. AGMC searches for 50 episodes and 100 episodes with a validation accuracy of $93.8 \%$ and $94.6 \%$, respectively. Thus, AGMC achieved a higher compression ratio with considerably fewer episodes.

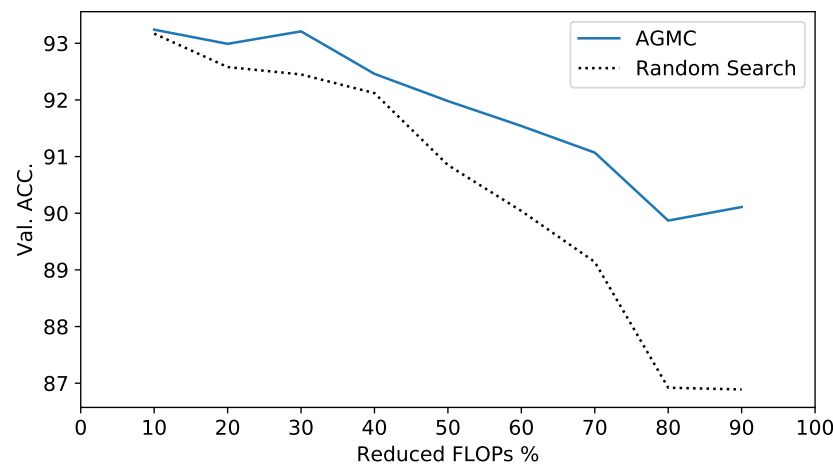

Figure 5. Validation accuracy comparison of random search and AGMC on ResNet-56 under different FLOPs constraints.

Additionally, we pruned ResNet-56 under different FLOPs-constraints. ResNet-56 contains 56 convolutional layers, which is far deeper and more challenging than ResNet-20 to prune. Figure 5 depicts the validation accuracy under different pruning ratios. In all cases, AGMC outperforms RS, as more FLOPs are pruned. For instance, with $10 \%$ FLOPs reduction, the performance of AGMC and RS are almost the same. However, with $90 \%$ FLOPs reduction, the AGMC surpasses the RS by a large margin.

Generalizability of the graph encoder. AGMC adopts a GCN-based graph encoder to embed DNNs topology structure. Since we model the DNNs as graphs under the same rule, the graph encoder trained on one DNN should also achieve similarly good performance on other similar DNNs. Thus, we investigated whether AGMC has learned the struc- 
tural pattern of ResNet-56 while performing channel pruning. We transferred the trained AGMC to ResNet-20, which is a similar network. When searching the pruning ratio of ResNet-20, we only updated the decoder parameters and did not require the graph encoder and RL agent's gradients. With 100 search episodes and 50\% FLOPs reduction on ResNet-20, the result of AGMC transferred from ResNet56 and obtained the validation accuracy of $92.08 \%$, which is similar to the AGMC trained on ResNet-20 with $94.6 \%$ validation accuracy.

\subsection{Over-parameterized DNN pruning}

We evaluate AGMC on ResNet-20/32/44/56/110 and VGG-16, often considered as over-parameterized DNNs. Such deep and compact networks involve billions of parameters, incurring high memory consumption. Thus, it is challenging to deploy them on edge devices with limited computing and power budgets.

We perform FLOPs-constrained pruning on overparameterized DNNs by leveraging the RL agent to search for pruning ratios for each convolutional layer. However, ResNet has residual connections, and different pruning ratios between residual connected layers will lead to featuremap dimension mismatch. To overcome this issue, we share the pruning ratio between the residual connection layers. Additionally, we follow the same experiment settings as in DSA [33] since it has a significant impact on the pruning results. For instance, the number of fine-tuning epochs is one of the key factors, where a larger value leads to higher test accuracy but with the cost of additional time and resources.

Table 1 reports the results of AGMC in comparison with existing pruning methods for over-parameterized networks. Our method outperforms the empirical policies [15, 24] by a large margin with $7.42 \%$ higher accuracy on ResNet20 and $4.36 \%$ on ResNet-56. Compared with the RLbased method, AMC [14], AGMC achieved 5.02\% and $2.56 \%$ higher accuracy on ResNet-20 and ResNet-56, respectively. Moreover, the networks pruned by AGMC yielded less accuracy loss compared with rule-based pruning methods [7, 28, 13, 33]. Additionally, we recorded the RL search time on ResNet-56 with 300 episodes. It takes $(320 \pm 30)$ seconds on RTX 8000 GPU to finish the entire search for the pruning ratios. For VGG-16 model trained on the ILSVRC-2012 dataset, we compared AGMC with handcrafted channel reduction methods (i.e., SPP [48], FP [24], and RNP [26]) and AMC [14]. Results show that AGMC outperformed all the baselines methods by a large margin.

\subsection{Mobile-friendly DNN pruning}

We further evaluated AGMC on mobile-friendly DNNs, such as MobileNet-v1/v2 [17, 39] and ShuffleNetv1/v2 [54, 29]. Instead of using standard convolutional layers, mobile-friendly DNNs have designed customized con-
Table 1. Pruning policy comparison of FLOPs-constrained compression on ResNet-20/32/44/56/110 and VGG-16 [44]. The ResNet family are trained on CIFAR-10 and VGG-16 is trained on the ImageNet (ILSVRC-2012) dataset.

\begin{tabular}{|c|c|c|c|c|}
\hline Model & Method & FLOPs & $\begin{array}{c}\text { Test } \\
\text { Acc. \% }\end{array}$ & $\Delta A c c$. \\
\hline \multirow{10}{*}{ ResNet20 } & Deep & $50 \%$ & 79.6 & -12.13 \\
\hline & Shallow & $50 \%$ & 83.2 & -8.53 \\
\hline & Uniform & $50 \%$ & 84 & -7.73 \\
\hline & SSL & $52 \%$ & 89.78 & -2.39 \\
\hline & MorphNet & $48 \%$ & 90.1 & -2.07 \\
\hline & Rethink & $60 \%$ & 91.07 & -1.34 \\
\hline & SFP & $58 \%$ & 90.83 & -1.37 \\
\hline & DSA & $50 \%$ & 91.38 & -0.79 \\
\hline & $\mathrm{AMC}$ & $50 \%$ & 86.4 & -5.33 \\
\hline & AGMC & $50 \%$ & 91.42 & -0.31 \\
\hline \multirow{8}{*}{ ResNet56 } & Uniform & $50 \%$ & 87.5 & -5.89 \\
\hline & Deep & $50 \%$ & 88.4 & -4.99 \\
\hline & SSL & $47 \%$ & 91.22 & -1.90 \\
\hline & MorphNet & $52 \%$ & 91.55 & -1.57 \\
\hline & Rethink & $50 \%$ & 93.07 & -0.73 \\
\hline & SFP & $50 \%$ & 92.26 & -1.33 \\
\hline & $\mathrm{AMC}$ & $50 \%$ & 90.2 & -3.19 \\
\hline & AGMC & $50 \%$ & 92.76 & -0.63 \\
\hline \multirow{5}{*}{ VGG-16 } & FP & $20 \%$ & 55.9 & -14.6 \\
\hline & RNP & $20 \%$ & 66.92 & -3.58 \\
\hline & SPP & $20 \%$ & 68.2 & -2.3 \\
\hline & $\mathrm{AMC}$ & $20 \%$ & 69.1 & -1.4 \\
\hline & AGMC & $20 \%$ & $\mathbf{7 0 . 3 5}$ & -0.15 \\
\hline \multirow{2}{*}{ ResNet110 } & $\mathrm{RS}$ & $50 \%$ & 87.26 & -6.42 \\
\hline & AGMC & $50 \%$ & 93.08 & -0.6 \\
\hline \multirow{2}{*}{ ResNet44 } & $\mathrm{RS}$ & $50 \%$ & 88.14 & -4.96 \\
\hline & AGMC & $50 \%$ & 92.28 & -0.82 \\
\hline \multirow{2}{*}{ ResNet32 } & RS & $50 \%$ & 89.57 & -3.06 \\
\hline & AGMC & $50 \%$ & 90.96 & -1.67 \\
\hline
\end{tabular}

volutional blocks to reduce the parameters, leading to better performance on edge devices. For instance, the MobileNetv1 block splits a traditional convolution into a pair of pointwise and depth-wise convolutions. Based on MobileNet-v1, MobileNet-v2 adds an additional linear expansion layer and introduced residual connections. To maintain the characteristics of the mobile-friendly DNNs, we have developed specific pruning strategies for them.

MobileNet-v1. The MobileNet-v1 block contains a depth-wise and a point-wise convolution, instead of pruning them separately, we view the two convolutions together and only prune point-wise convolutions. Since the depthwise convolution only operates on one input channel and 
Table 2. Pruning policy comparison of FLOPs-constrained compression on MobileNet-v1/v2 and ShuffleNet-v1/v2. The MobileNet-v1/v2 are trained on the ImageNet (ILSVRC-2012) dataset, and the ShuffleNet-v1/v2 are trained on the CIFAR-100 dataset. The column FLOPs denotes the ratio between the FLOPs of the compressed model and the original model.

\begin{tabular}{|l|c|l|l|l|}
\hline Model & Method & FLOPs & $\begin{array}{l}\text { Test } \\
\text { Acc. }\end{array}$ & $\begin{array}{l}\Delta \text { Acc. } \\
\%\end{array}$ \\
\hline \hline \multirow{4}{*}{ MobileNet-v1 } & uniform[17] & $56 \%$ & 68.10 & -2.5 \\
& uniform[17] & $41 \%$ & 66.90 & -3.7 \\
& AMC[14] & $40 \%$ & 68.90 & -1.7 \\
& AGMC & $\mathbf{4 0 \%}$ & $\mathbf{6 9 . 4 0}$ & $\mathbf{- 1 . 2}$ \\
\hline \multirow{3}{*}{ MobileNet-v2 } & uniform [39] & & 69.80 & -2 \\
& AMC[14] & $70 \%$ & 70.80 & -1 \\
& AGMC & & $\mathbf{7 0 . 8 7}$ & $\mathbf{- 0 . 9 3}$ \\
\hline \multirow{2}{*}{ ShuffleNet-v1 } & RS & $60 \%$ & 63.70 & -4.94 \\
& AGMC & $60 \%$ & $\mathbf{6 5 . 2 6}$ & $\mathbf{- 3 . 3 8}$ \\
\hline \hline \multirow{2}{*}{ ShuffleNet-v2 } & RS & $60 \%$ & 65.74 & -3.11 \\
& AGMC & $60 \%$ & $\mathbf{6 6 . 2 8}$ & $\mathbf{- 2 . 5 7}$ \\
\hline
\end{tabular}

pruning that filter will cause information loss for the corresponding channel.

MobileNet-v2. Similar to MobileNet-v1, we prune linear expansion layers and point-wise convolutional layers. Since residual connections are between linear expansion layers, we share the linear expansion layers' pruning ratio.

ShuffleNet-v1/v2. ShuffleNet uses blocks containing depth-wise and point-wise convolutions, channel shuffle, linear expansion, and residual connections. To avoid dimension mismatch when downsampling, we consider the ShuffleNet blocks together and perform channel pruning inside the blocks. In a ShuffleNet block, we do not prune the expansion layer (the output layer of the block) and only prune the point-wise filters.

The results for mobile-friendly networks are given in Table 2. On MobileNet-v1/v2, we compare AGMC with the uniform pruning policy and the RL-based method AMC. Compared to the uniform policy, which sets the compression ratio uniformly, AGMC achieves a higher compression ratio with only $1.2 \%$ test accuracy loss. Furthermore, our efficient layer embedding outperforms AMC on both MobileNet-v1 and MobileNet-v2, with the same target FLOPs. Similarly, our method succeeded in pruning $40 \%$ of the ShuffleNet-v1/v2 FLOPs and obtaining a more accurate compressed model than random search.

\subsection{Inference acceleration and memory saving}

Here, we discuss the inference speed of the compressed ResNet-20/56, VGG-16, and MobileNet-v1 on an Nvidia RTX 2080Ti GPU. AGMC performs channel pruning on
Table 3. Latency and GPU memory usage of pruned models using AGMC. We analyzed ResNet-20/56 on CIFAR-10 and VGG-16 and MobileNet-v1 on ImageNet dataset.

\begin{tabular}{|l|c|c|c|}
\hline Model & FLOPs & Latency & GPU Mem. \\
\hline \hline \multirow{2}{*}{ MobileNet-v1 } & $100 \%$ FLOPs & $11.02 \mathrm{~ms}$ & $17 \mathrm{MB}$ \\
& $40 \%$ FLOPs & $10.52 \mathrm{~ms}$ & $14 \mathrm{MB}$ \\
\hline \multirow{2}{*}{ VGG-16 } & $100 \%$ FLOPs & $20.52 \mathrm{~ms}$ & $528 \mathrm{MB}$ \\
& $20 \%$ FLOPs & $16.82 \mathrm{~ms}$ & $387 \mathrm{MB}$ \\
\hline \multirow{2}{*}{ ResNet-56 } & $100 \%$ FLOPs & $0.52 \mathrm{~ms}$ & $3.4 \mathrm{MB}$ \\
& $50 \%$ FLOPs & $0.48 \mathrm{~ms}$ & $1.8 \mathrm{M}$ \\
\hline \multirow{2}{*}{ ResNet-20 } & $100 \%$ FLOPs & $0.32 \mathrm{~ms}$ & $1.1 \mathrm{MB}$ \\
& $50 \%$ FLOPs & $0.30 \mathrm{~ms}$ & $565 \mathrm{~KB}$ \\
\hline
\end{tabular}

convolutional layers, accelerating the inference on parallel devices like GPUs. We calculated the inference speed of the pruned models and compared them with the original model. We used batch size 32, and the compressed models are tested on CIFAR-10 and ILSVRC-2012 datasets. As shown in Table 3, the models pruned by AGMC achieve notable GPU memory reduction. For example, for VGG-16, the original model's GPU memory usage is $528 \mathrm{MB}$, since it has dense layers and its first dense layer contains 25088 neurons. The $20 \%$ FLOPs VGG-16 with pruned convolutional layers significantly reduced the feature map size input to dense layers, taking $141 \mathrm{MB}$ memory less than the original. Moreover, without losing too much test accuracy, all the models pruned by AGMC achieved remarkable inference speedup. For instance, the 20\% FLOPs VGG-16 achieved $1.22 \times$ speedup on the ImageNet dataset.

\section{Conclusion}

This paper proposed an Auto Graph encoder-decoder Model Compression (AGMC), which combines graph convolutional networks and reinforcement learning to explore network compression policies automatically. To the best of our knowledge, this is the first work to model DNNs as computational graphs to enhance model compression. Furthermore, we conducted comprehensive experiments on over-parameterized and mobile-friendly DNNs. In the experiment, we show the superiority of our learning-based DNN embedding. By learning DNNs' embedding from their structure information, AGMC outperforms all the rulebased DNN embedding methods by a large margin. On over-parameterized, such as ResNet-56, our method defeats all the baselines with only $0.63 \%$ accuracy loss. Additionally, AGMC successfully compressed mobile-friendly DNNs, which are already compact. For instance, in the MobileNet-V1, we achieve a higher compression ratio than baselines with only $1.2 \%$ accuracy loss. 


\section{References}

[1] Martin Abadi, Paul Barham, Jianmin Chen, Zhifeng Chen, Andy Davis, Jeffrey Dean, Matthieu Devin, Sanjay Ghemawat, Geoffrey Irving, Michael Isard, Manjunath Kudlur, Josh Levenberg, Rajat Monga, Sherry Moore, Derek G. Murray, Benoit Steiner, Paul Tucker, Vijay Vasudevan, Pete Warden, Martin Wicke, Yuan Yu, and Xiaoqiang Zheng. Tensorflow: A system for large-scale machine learning. In 12th USENIX Symposium on Operating Systems Design and Implementation (OSDI 16), pages 265-283, 2016. 1

[2] Cody Blakeney, Yan Yan, and Ziliang Zong. Is pruning compression?: Investigating pruning via network layer similarity. WACV, pages 903-911, 2020. 2

[3] Stephen Boyd, Neal Parikh, Eric Chu, Borja Peleato, and Jonathan Eckstein. Distributed optimization and statistical learning via the alternating direction method of multipliers. Found. Trends Mach. Learn., 3(1):1-122, Jan. 2011. 2

[4] Yukang Chen, Gaofeng Meng, Qian Zhang, Shiming Xiang, Chang Huang, Lisen Mu, and Xinggang Wang. Renas - reinforced evolutionary neural architecture search. $C V P R$, pages 4787-4796, 2019. 2

[5] Lukasz Dudziak, Thomas Chau, Mohamed S. Abdelfattah, Royson Lee, Hyeji Kim, and Nicholas D. Lane. BRP-NAS: Prediction-based NAS using gcns. 2021. 2

[6] Yang Gao, Hong Yang, Peng Zhang, Chuan Zhou, and Yue Hu. Graphnas: Graph neural architecture search with reinforcement learning. arXiv: Learning, 2019. 2

[7] Ariel Gordon, Elad Eban, Ofir Nachum, Bo Chen, Hao Wu, Tien-Ju Yang, and Edward Choi. MorphNet: Fast \& Simple Resource-Constrained Structure Learning of Deep Networks. In 2018 IEEE/CVF Conference on Computer Vision and Pattern Recognition, pages 1586-1595, Salt Lake City, UT, June 2018. IEEE. 5, 7

[8] Yong Guo, Yin Zheng, Mingkui Tan, Qi Chen, Jian Chen, Peilin Zhao, and Junzhou Huang. NAT: Neural architecture transformer for accurate and compact architectures. In Proc. of the Advances in Neural Information Processing Systems, volume 32, pages 737-748. Curran Associates, Inc., 2019. 2

[9] Song Han, Junlong Kang, Huizi Mao, Yiming Hu, Xin Li, Yubin Li, Dongliang Xie, Hong Luo, Song Yao, Yu Wang, Huazhong Yang, and (Bill) J. William Dally. ESE: Efficient speech recognition engine with sparse LSTM on FPGA. Proc. of the ACM/SIGDA International Symposium on FieldProgrammable Gate Arrays, pages 75-84, 2017. 2

[10] Song Han, Huizi Mao, and J. William Dally. Deep compression: Compressing deep neural network with pruning, trained quantization and huffman coding. International conference on learning representations, 2015. 1, 2

[11] Babak Hassibi and G. David Stork. Second order derivatives for network pruning: Optimal brain surgeon. NIPS, pages 164-171, 1992. 1, 2

[12] Kaiming He, Xiangyu Zhang, Shaoqing Ren, and Jian Sun. Deep residual learning for image recognition. pages 770 778, 2016. 2, 3, 4, 5

[13] Yang He, Guoliang Kang, Xuanyi Dong, Yanwei Fu, and Yi Yang. Soft Filter Pruning for Accelerating Deep Convolutional Neural Networks. In Proceedings of the Twenty-
Seventh International Joint Conference on Artificial Intelligence, pages 2234-2240, Stockholm, Sweden, July 2018. International Joint Conferences on Artificial Intelligence Organization. 7

[14] Yihui He, Ji Lin, Zhijian Liu, Hanrui Wang, Li-Jia Li, and Song Han. Amc: Automl for model compression and acceleration on mobile devices. In Proc. of the European Conference on Computer Vision (ECCV), pages 784-800, 2018. 1, 2, 4, 5, 7, 8

[15] Yihui He, Xiangyu Zhang, and Jian Sun. Channel pruning for accelerating very deep neural networks. In Proc. of the IEEE International Conference on Computer Vision, pages 1389-1397, 2017. 2, 5, 7

[16] E. Geoffrey Hinton, Oriol Vinyals, and Jeffrey Dean. Distilling the knowledge in a neural network. CoRR, 2015. 1, 2

[17] Andrew G. Howard, Menglong Zhu, Bo Chen, Dmitry Kalenichenko, Weijun Wang, Tobias Weyand, Marco Andreetto, and Hartwig Adam. Mobilenets: Efficient convolutional neural networks for mobile vision applications. CoRR, abs/1704.04861, 2017. 2, 5, 7, 8

[18] Gao Huang, Shichen Liu, Laurens Van der Maaten, and Kilian Q Weinberger. Condensenet: An efficient densenet using learned group convolutions. In Proc. of the IEEE conference on computer vision and pattern recognition, pages 2752-2761, 2018. 2

[19] Benoit Jacob, Skirmantas Kligys, Bo Chen, Menglong Zhu, Matthew Tang, Andrew Howard, Hartwig Adam, and Dmitry Kalenichenko. Quantization and training of neural networks for efficient integer-arithmetic-only inference. In Proc. of the IEEE Conference on Computer Vision and Pattern Recognition, pages 2704-2713, 2018. 1, 2

[20] Houxiang Ji, Linghao Song, Li Jiang, Hai Halen Li, and Yiran Chen. Recom: An efficient resistive accelerator for compressed deep neural networks. In Proc of. Design, Automation \& Test in Europe Conference \& Exhibition (DATE), pages 237-240. IEEE, 2018. 2

[21] Thomas N Kipf and Max Welling. Semi-supervised classification with graph convolutional networks. arXiv preprint arXiv:1609.02907, 2016. 1

[22] Thomas N. Kipf and Max Welling. Semi-supervised classification with graph convolutional networks. In Proc. of the International Conference on Learning Representations (ICLR), 2017. 2

[23] A Krizhevsky and G Hinton. Learning multiple layers of features from tiny images. 2009. 5

[24] Hao Li, Asim Kadav, Igor Durdanovic, Hanan Samet, and Hans Peter Graf. Pruning filters for efficient convnets. CoRR, abs/1608.08710, 2016. 2, 5, 6, 7

[25] Timothy P Lillicrap, Jonathan J Hunt, Alexander Pritzel, Nicolas Heess, Tom Erez, Yuval Tassa, David Silver, and Daan Wierstra. Continuous control with deep reinforcement learning. arXiv preprint arXiv:1509.02971, 2015. 1, 4

[26] Ji Lin, Yongming Rao, Jiwen Lu, and Jie Zhou. Runtime neural pruning. In Proc. of the Advances in Neural Information Processing Systems, pages 2181-2191, 2017. 2, 5, 7 
[27] Ning Liu, Xiaolong Ma, Zhiyuan Xu, Yanzhi Wang, Jian Tang, and Jieping Ye. Autocompress: An automatic dnn structured pruning framework for ultra-high compression rates. In Proc. of Artificial Intelligence Conference (AAAI), pages 4876-4883, 2020. 1, 2, 5

[28] Zhuang Liu, Mingjie Sun, Tinghui Zhou, Gao Huang, and Trevor Darrell. Rethinking the value of network pruning. International Conference on Learning Representations (ICLR), 2019. 5,7

[29] Ningning Ma, Xiangyu Zhang, Hai-Tao Zheng, and Jian Sun. Shufflenet v2: Practical guidelines for efficient cnn architecture design. In Proc. of the European conference on computer vision (ECCV), pages 116-131, 2018. 2, 5, 7

[30] Rahim Mammadli, Felix Wolf, and Ali Jannesari. The art of getting deep neural networks in shape. volume 15, pages 62:1-62:21, Jan. 2019. 2

[31] Sachin Mehta, Hannaneh Hajishirzi, and Mohammad Rastegari. Dicenet: Dimension-wise convolutions for efficient networks. IEEE Transactions on Pattern Analysis and Machine Intelligence, 2020. 2

[32] Pavlo Molchanov, Arun Mallya, Stephen Tyree, Iuri Frosio, and Jan Kautz. Importance estimation for neural network pruning. In Proceedings of the IEEE Conference on Computer Vision and Pattern Recognition, pages 11264-11272, 2019. 1, 2

[33] Xuefei Ning, Tianchen Zhao, Wenshuo Li, Peng Lei, Yu Wang, and Huazhong Yang. DSA: More Efficient Budgeted Pruning via Differentiable Sparsity Allocation. In Andrea Vedaldi, Horst Bischof, Thomas Brox, and Jan-Michael Frahm, editors, European Conference on Computer Vision ECCV 2020, volume 12348, pages 592-607, Cham, 2020. Springer International Publishing. Series Title: Lecture Notes in Computer Science. 5, 7

[34] Wonpyo Park, Dongju Kim, Yan Lu, and Minsu Cho. Relational knowledge distillation. In Proc. of the IEEE Conference on Computer Vision and Pattern Recognition, pages 3967-3976, 2019. 1, 2

[35] Adam Paszke, Sam Gross, Francisco Massa, Adam Lerer, James Bradbury, Gregory Chanan, Trevor Killeen, Zeming Lin, Natalia Gimelshein, Luca Antiga, Alban Desmaison, Andreas Kopf, Edward Yang, Zachary DeVito, Martin Raison, Alykhan Tejani, Sasank Chilamkurthy, Benoit Steiner, Lu Fang, Junjie Bai, and Soumith Chintala. Pytorch: An imperative style, high-performance deep learning library. In H. Wallach, H. Larochelle, A. Beygelzimer, F. d'Alché-Buc, E. Fox, and R. Garnett, editors, Advances in Neural Information Processing Systems, volume 32, pages 8026-8037. Curran Associates, Inc., 2019. 1

[36] Antonio Polino, Razvan Pascanu, and Dan Alistarh. Model compression via distillation and quantization. ICLR, 2018. 1,2

[37] Olga Russakovsky, Jia Deng, Hao Su, Jonathan Krause, Sanjeev Satheesh, Sean Ma, Zhiheng Huang, Andrej Karpathy, Aditya Khosla, Michael Bernstein, Alexander C. Berg, and Li Fei-Fei. ImageNet Large Scale Visual Recognition Challenge. International Journal of Computer Vision (IJCV), 115(3):211-252, 2015. 5
[38] T. N. Sainath, B. Kingsbury, V. Sindhwani, E. Arisoy, and B. Ramabhadran. Low-rank matrix factorization for deep neural network training with high-dimensional output targets. In 2013 IEEE International Conference on Acoustics, Speech and Signal Processing, pages 6655-6659, 2013. 1, 2

[39] Mark Sandler, G. Andrew Howard, Menglong Zhu, Andrey Zhmoginov, and Liang-Chieh Chen. MobileNetV2: Inverted residuals and linear bottlenecks. pages 4510-4520, 2018. 2, $5,7,8$

[40] Michael Schlichtkrull, Thomas N. Kipf, Peter Bloem, Rianne van den Berg, Ivan Titov, and Max Welling. Modeling relational data with graph convolutional networks. In Aldo Gangemi, Roberto Navigli, Maria-Esther Vidal, Pascal Hitzler, Raphaël Troncy, Laura Hollink, Anna Tordai, and Mehwish Alam, editors, The Semantic Web, pages 593-607, Cham, 2018. Springer International Publishing. 2

[41] John Schulman, Filip Wolski, Prafulla Dhariwal, Alec Radford, and Oleg Klimov. Proximal policy optimization algorithms, 2017. 4

[42] Han Shi, Renjie Pi, Hang Xu, Zhenguo Li, James T. Kwok, and Tong Zhang. Bridging the gap between sample-based and one-shot neural architecture search with BONAS. 2020. 2

[43] David Silver, Guy Lever, Nicolas Heess, Thomas Degris, Daan Wierstra, and Martin Riedmiller. Deterministic policy gradient algorithms. volume 1, 06 2014. 1

[44] Karen Simonyan and Andrew Zisserman. Very deep convolutional networks for large-scale image recognition. international conference on learning representations, 2015. 2, 5, 7

[45] S. Richard Sutton, A. David Mcallester, P. Satinder Singh, and Yishay Mansour. Policy gradient methods for reinforcement learning with function approximation. Neural Information Processing Systems, pages 1057-1063, 1999. 1

[46] Sridhar Swaminathan, Deepak Garg, Rajkumar Kannan, and Frederic Andres. Sparse low rank factorization for deep neural network compression. Neurocomputing, pages 185-196, 2020. 1, 2

[47] Sheng Kai Tai, Richard Socher, and D. Christopher Manning. Improved semantic representations from treestructured long short-term memory networks. International Workshop on the ACL2 Theorem Prover and Its Applications, 2015. 3

[48] Huan Wang, Qiming Zhang, Yuehai Wang, and Roland Hu. Structured probabilistic pruning for deep convolutional neural network acceleration. British Machine Vision Conference, 2017. 2, 5,7

[49] J. Wang, H. Bai, J. Wu, and J. Cheng. Bayesian automatic model compression. IEEE Journal of Selected Topics in Signal Processing, 14(4):727-736, 2020. 1, 2

[50] Wei Wen, Chunpeng Wu, Yandan Wang, Yiran Chen, and Hai Li. Learning Structured Sparsity in Deep Neural Networks. In Advances in Neural Information Processing Systems, volume 29. Curran Associates, Inc., 2016. 5

[51] Xia Xiao, Zigeng Wang, and Sanguthevar Rajasekaran. Autoprun: Automatic network pruning by regularizing auxiliary parameters. Advances in Neural Information Processing Systems (NIPS), pages 13681-13691, 2019. 2 
[52] Keyulu Xu, Weihua Hu, Jure Leskovec, and Stefanie Jegelka. How powerful are graph neural networks? In Proc. of International Conference on Learning Representations (ICLR), 2019. 1

[53] Muhan Zhang and Yixin Chen. Link prediction based on graph neural networks. In Advances in Neural Information Processing Systems, pages 5165-5175, 2018. 1

[54] Xiangyu Zhang, Xinyu Zhou, Mengxiao Lin, and Jian Sun. Shufflenet: An extremely efficient convolutional neural network for mobile devices. In Proc. of the IEEE conference on computer vision and pattern recognition, pages 6848-6856, 2018. 2, 5, 7

[55] Barret Zoph and V. Quoc Le. Neural architecture search with reinforcement learning. international conference on learning representations, 2017. 2 